\title{
Fission and Fusion Nuclear Reactions Second Law Analyses Based on Exergy Method
}

\author{
P. Palazzo* \\ Sapienza Università di Roma, Department of Astronautical Electrical and Energy Engineering (DIAEE), \\ 00184 Roma, Italy \\ E-mail: ${ }^{1}$ pierfrancesco.palazzo@ uniroma1.it
}

Received 09 August 2018, Revised 14 October 2018, Accepted 21 October 2018

\begin{abstract}
The present research aims at specializing the second law analysis to characterize balances of properties, and efficiencies of processes, occurring in nuclear reactions. The conceptual schema is underpinned by the paradigm of microscopic few-particle systems and the inter-particle kinetic energy and binding potential energy determined by interactions among atomic nuclei and subatomic particles in non-equilibrium states along irreversible phenomena. The definition here proposed for thermodynamic entropy calculation is based on energy and exergy both being measurable properties used to directly derive entropy variations determined by elemental fission and fusion nuclear reactions in operating industrial plants.
\end{abstract}

Keywords: Nonequilibrium; first law; second law; generalized thermodynamic entropy; generalized exergy; fission nuclear reaction; fusion nuclear reaction.

\section{Introduction}

The interest in developing second law analysis founded on exergy method applied to fission and fusion nuclear reactions can be found in: i) the observation of physical phenomena and nuclear processes through a thermodynamic standpoint; ii) the need of adopting a more extended approach to nuclear reactors design in the perspective of the optimization and confrontation of different types of technologies and plant configurations. The exergy method focuses on irreversible phenomena associated to nonequilibrium states and processes occurring in all macroscopic and microscopic physical systems. The degree of irreversibility of non-equilibrium processes is correlated to the amount of exergy destruction representing the indicator of the degree of dissipation of all energy and interaction forms. For these reasons, it is worth applying this method to nuclear reactions with the aim of extending the overall plant mass and energy balances and evaluate exergy efficiencies. To do so, an analysis of concepts underpinning the definitions of thermodynamic entropy and exergy properties is here summarized to demonstrate their implications in nuclear physics.

Usually, second law analyses consider the conventional plant or the nuclear reactor or both jointly combined to achieve an overall balance. As far as light water boiling technology and Boiling Water Reactors (BWR) power units are concerned, a study was carried out by Dunbar et al. on an operating plant [1]. As regards light water pressurized technology, more recent studies are reported in the literature for Pressurized Water Reactors (PWR) [2] and Multipurpose Advanced Reactor inherently Safe (MARS) [3]. Despite the accuracy of those studies, no specific mention is provided on the elemental nuclear reaction and the implication on the behavior and performance of plant core. Though, here the purpose is to bridge this gap with the analysis focusing on the elementary fission or fusion nuclear reaction representing the crucial process stage occurring in the core of fission power plants or in the plasma chamber of ITER (under construction) or DEMO (under design) fusion reactors.

As regard the nuclear physical aspect, the literature reports studies focusing on the exergy analysis of nuclear radiation along decay processes [4,5]. In particular, classical and quantum statistical methods have been adopted to evaluate exergy based on barions motion and momentum to achieve exergy fluxes calculated by means of physical parameters characterizing the particles motion $[4,5]$. These evaluations are underpinned by statistical models to describe nuclear fuel bulk reactions occurring in operational industrial plants. The present research focuses on the thermodynamic processes occurring within the core of reactors. Here, the intent is to analyze the elemental fission and fusion reactions and to propose an alternative method based on binding potential energy among protons and neutrons, calculated on the basis of mass defect in different physical states [6]. The objective is to evaluate the thermodynamic state and process variables governing nuclear reactions and to provide the bases to achieve a complete evaluation of plant configurations and a confrontation among different technologies. This perspective allows to assess the balance and efficiency that should be accounted for in an overall Second Law analysis based on exergy method here adopted to provide an operative tool for calculations in applications and reactors design. To do so, Second Law analyses need to be carried out adopting measurable properties and for this very reason the exergy method, and the formulation of thermodynamic entropy defined by Hatsopoulos, Gyftopoulos and Beretta will be adopted as described in the following sections. 


\section{Second Law Analysis and Exergy Method Applied to Nuclear Reactions}

Nuclear reactions can be regarded as elemental nonequilibrium irreversible processes occurring in few-particle systems constituted by nuclei, neutrons and other subnuclear particles interacting because of collisions occurring at defined thermodynamic conditions. The definition of entropy property of a system $A$, beside the classical formulation of Clausius $S=\frac{\delta Q}{T}$, has been expressed by Hatsopoulos, Gyftopoulos and Beretta in the following form [7-11]:

$S_{1}-S_{0}=\frac{1}{C_{R}}\left[\left(E_{1}-E_{0}\right)-\left(\Omega_{1}^{R}-\Omega_{0}^{R}\right)\right]$

where $C_{R}$ is a constant characterizing an external reference system $R$ behaving as a reservoir, $E$ is the internal energy determined by the temperature associated to the kinetic energy of interacting particles and reaction products, and $\Omega^{R}$ is the available energy of the system interacting with

the reservoir. The literature reports the proof that $C_{R}=T_{R}$ [7]. Moreover, it has been proved that entropy is an inherent property of any system, large or small, in any state, equilibrium or non-equilibrium [8,9] and, for this reason, the reservoir behaves as an auxiliary system only [4]. The definition of thermal entropy can be expressed in the form:

$$
S_{1}^{T}-S_{0}^{T}=\frac{1}{T_{R}}\left[\left(E_{1}-E_{0}\right)-\left(E X_{1}^{R}-E X_{0}^{R}\right)\right]^{T}
$$

where the available energy $\Omega^{R}$ is replaced by the thermal exergy $E X^{R}$. This expression has been proved [7] by means of the concept of non-existence of Perpetual Motion Machine of the Second Kind (PMM2). Indeed, the non-existence of PMM2 implies the impossibility of a complete thermalmechanical conversion of thermal energy into work thus meaning that the energy conversion efficiency $\eta^{T}<1$. This logical inference can be replicated for nuclear-mechanical conversion of mass into work where mass interaction is associated to the potential energy, or binding energy, among particles constituting a system. Hence, the definition of nuclear entropy can be assumed to be:

$$
S_{1}^{N}-S_{0}^{N}=\frac{1}{\mu_{R}^{N}}\left[\left(E_{1}^{N}-E_{0}^{N}\right)-\left(E X_{1}^{R}-E X_{0}^{R}\right)\right]^{N}
$$

where $C_{R}=\mu_{R}^{N}$ and $\mu_{R}^{N}$ is the reference nuclear potential of the reservoir and $E^{N}$ is the binding potential energy associated to strong interactions among nucleons. The method here adopted is founded on the binding energies calculated in terms of mass defects before and after a nuclear reaction according to the approach already used and reported in the literature by Badescu and Isvoranu [4,5] and by Hermann [6]. The Figure 1 describes the curves of binding potential energy for nuclei involved in fission and fusion reactions respectively.

In this particular case, the non-existence of PMM2 implies the impossibility of a complete nuclear-mechanical conversion of nuclear potential into work thus meaning that the energy conversion efficiency $\eta^{N}<1$.

Finally, the mechanical entropy $S^{M}$ accounts for the density of: (i) interparticle kinetic energy correlated to the frequency of collisions, and (ii) the potential energy correlated to the intensity of actions both depending on the volume. Hence, the following expression:

$$
S_{1}^{M}-S_{0}^{M}=\frac{\bar{R}}{P_{R} V_{R}}\left[\left(E_{1}-E_{0}\right)-\left(E X_{1}^{R}-E X_{0}^{R}\right)\right]^{M}
$$

in which $\frac{1}{C_{R}}=\frac{\bar{R}}{P_{R} V_{R}}$, constitutes an additional term of the generalized thermodynamic entropy suitable to characterize the mechanical internal energy $P V$ associated to pressure and volume. As entropy is an additive property, the generalized definition is obtained by the sum of its thermal, nuclear and mechanical components: $S^{G}=S^{T}+S^{N}+S^{M}$

[12-13].

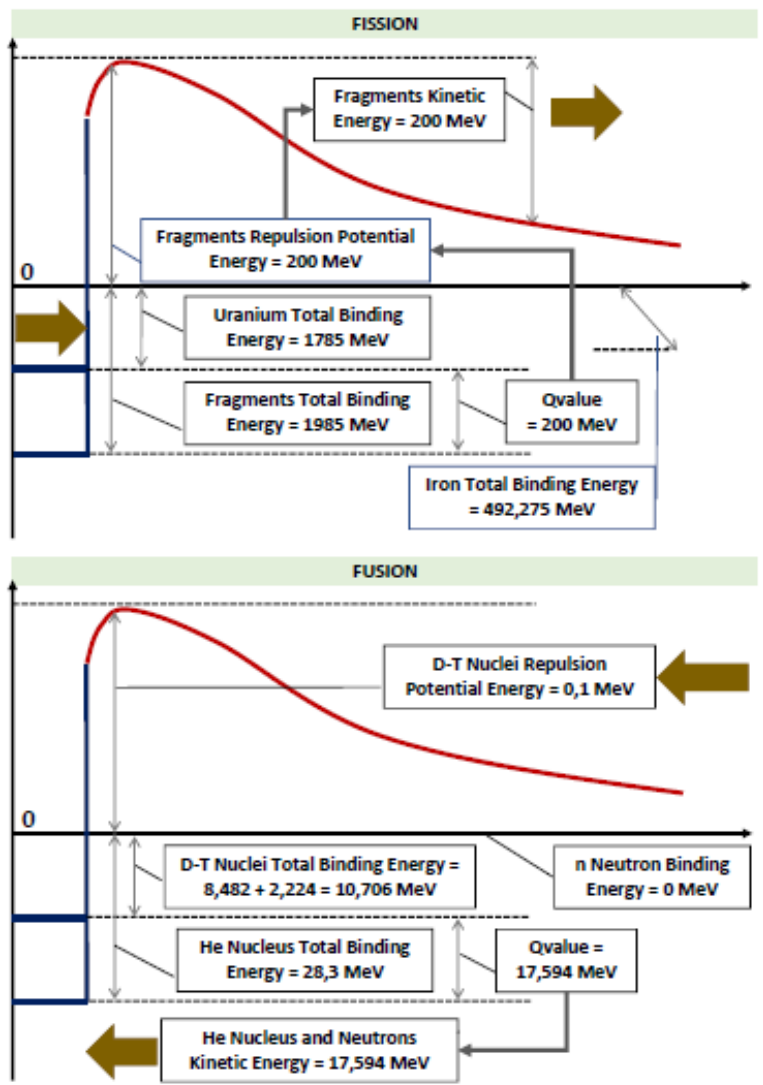

Figure 1. Binding potentials in fission and fusion nuclear reactions. The horizontal $x$-axis represents the distance between interacting particles, the vertical y-axis represents the inter-particle energy.

With the definitions of entropy related to exergy, and being $A R$ the composite of mutually interacting system $A$ and reservoir $R$, the generalized exergy balance of an individual fission or fusion nuclear reaction can be expressed by means of different exergy contributions. They are thermal exergy producing available work interaction $E X^{T}=\left(W_{10}^{A R \rightarrow}\right)_{H E A T}^{M A X}$, nuclear exergy producing work interaction 
$E X^{N}=\left(W_{10}^{A R \rightarrow}\right)_{M A S S}^{M A X}$, mechanical exergy producing heat interaction $E X^{M}=\left(Q_{10}^{A R \rightarrow}\right)_{\text {WORK }}^{M A X}$ mechanical exergy producing mass interaction $E X^{M}=\left(M_{10}^{A R \rightarrow}\right)_{W O R K}^{M A X}$; therefore, the expression of generalized exergy is the following [14]:

$$
\begin{aligned}
& E X_{\text {NUCLEAR }}^{G}=E X^{T}+E X^{N}+E X^{M} \\
& =\left(W_{10}^{A R \rightarrow}\right)_{\text {HEAT }}^{M A X}+\left(W_{10}^{A R \rightarrow}\right)_{M A S S}^{M A X} \\
& +\left(Q_{10}^{A R \rightarrow}\right)_{\text {WORK }}^{M A X}+\left(M_{10}^{A R \rightarrow}\right)_{\text {WORK }}^{M A X}
\end{aligned}
$$

This definition of generalized exergy of nuclear reactions is underpinned, on the one side, by the concept of equivalence between heat interaction and work interaction and the equivalence between mass interaction and work interaction; on the other side, the inter-convertibility of heatto-work and work-to-heat as well as the inter-convertibility of mass-to-work and work-to-mass is a counter-proof of the equivalence above mentioned [15-18]. It is worth using the expressions above discussed for nuclear processes since energy and exergy are measurable properties. Moreover, the thermodynamic state of reference system behaving as a reservoir, can be arbitrarily selected being an auxiliary system only in the definition of thermodynamic entropy [810] and its components [14]. Hence, the calculation of entropy can be directly derived once thermodynamic states before and after nuclear reactions are defined. This method, usually adopted for many-particle systems and properties describing macroscopic systems in plant optimization [19], will be here specially applied to elemental fission and fusion processes as demonstrated in the following sections.

\section{Fission Nuclear Reaction Exergy Analysis}

A typical Uranium ${ }_{92} U^{235}$ fission reaction is represented by [20-21]:

$$
\begin{aligned}
& { }_{92} U^{235}+{ }_{0} n^{1} \rightarrow_{92} U^{236^{*}} \\
& \rightarrow{ }_{Z 1} X^{A 1}+{ }_{Z 2} Y^{A 2}+2,43_{0} n^{1}+\text { Qvalue }
\end{aligned}
$$

Before the fission occurs, strong attraction interactions among nucleons behave as a constraint and hold the nucleus as a unique whole system. The nucleus fission produces fragments ${ }_{Z 1} X^{A 1},{ }_{Z 2} Y^{A 2}$ composed by nuclei of lower atomic number, with respect to the uranium atomic number, neutrons and gamma radiation. The increase of particles mass dispersion and interactions distribution among fragments causes the transition from a concentrated mass, characterized by a unique position and velocity, to a subdivided configuration. This transition implies that positions are dispersed and velocities distributed with random spectra compared with the initial nucleus, due to the intrinsically probabilistic statistical and quantum nature of phenomena at microscopic nuclear and sub-nuclear level. Once the inter-nucleons attraction bond barrier has been overcome during the fission, fragments move accelerating away from each other due to electro-magnetic repulsion interactions. The repulsion potential energy existing immediately after the fission is transformed into fragments kinetic energy released to the surrounding fuel bulk and the fission temperature is decreased to the fuel rods temperature. The fission temperature is conventionally calculated considering the relation $k_{B} T=210 \mathrm{MeV}$ where $k_{B}=8,617 \times 10^{-11} \mathrm{MeV} / \mathrm{K}$ is the Boltzmann constant and $\mathrm{MeV}=1,602 \cdot 10^{-13} \mathrm{~J}$.

This temperature difference is not used in a conventional industrial reactor to produce work interaction hence it is irreversibly dissipated. The reaction is not reversible because the heat interaction amount released is not sufficient to reverse back the nucleus to its original configuration thus requiring an inverse fusion reaction with heat input. In fact, the fission determines the stochastic distribution of velocity vectors pertaining to each and every fission fragment in such a way to prevent the occurrence of any reversible process back to the initial unique mass state with energy associated to the nucleus center of mass. Thus, nucleus fission into fragments increases the degree of velocity distribution and position dispersion among all particles, hence increases the entropy property characterizing the thermodynamic state change after fission process. The entity of entropy increase caused by the two different contributions above identified is the objective of the following analysis.

The definition of generalized thermodynamic entropy $S^{G}$ variation, outlined in the previous section and here adopted, requires the calculation of the generalized exergy $E X^{G}$ variation that can be expressed in the following terms taking into account the contribution balance of all components:

$$
\begin{aligned}
& \underset{\text { FISSION }}{\Delta E X_{\text {NUCLAR }}^{G}}=\left(\Delta E X^{M}+\Delta E X^{T}+\Delta E X^{N}\right)_{\text {NUCLEAR }} \\
& =-\left[\left(E X^{M}+E X^{T}+E X^{N}\right)^{\text {BEISSION }}\right] \\
& -\left[-\left(E X^{M}+E X^{T}+E X^{N}\right) \text { FISTION }\right]
\end{aligned}
$$

Before the fission, the mechanical exergy $E X^{M}$ consists of the capability, of colliding neutron and target nucleus kinetic energy, to be converted into thermal energy along a reversible process, however for moderated neutrons this component is negligible. The thermal exergy $E X^{T}$ related to the fuel bulk temperature characterizing target nucleus with respect to the environment, is negligible. The nuclear exergy $E X^{N}$ associated to the nucleus binding potential energy, is equal to the sum of inter-nucleons strong attraction potential energy and inter-nucleon electro-magnetic repulsion

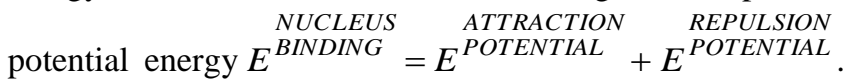
This resulting potential energy can be theoretically transferred as maximum net useful work interaction along a mass-to-work direct conversion cycle with efficiency $\eta^{N}$.

Hence, only nuclear exergy is available before fission. The total binding potential energy of one nucleus of uranium 
${ }_{92} U^{235}$ can be assumed equal to about $1785.5 \mathrm{MeV}$ [20] and constitutes the theoretical available potential energy $E^{\text {NINDING }}$ that the nucleus can release in the form of repulsion potential energy among all nucleons.

After the fission, fragments with lower atomic number and atomic mass are generated. The initial nucleus binding potential energy of the starting whole nucleus is transformed into two contributions constituted by: i) fragments binding potential energy, and ii) repulsion potential energy in turn transformed into fragments kinetic energy expressed as Qvalue representing the useful energy released along the fission reaction. Hence, thermal energy only is available that can be converted into mechanical energy and transferred as work interaction to the external system along a heat-to-work conversion direct cycle with efficiency $\eta^{T}$. This implies that in turn, thermal exergy only is available after fission.

The overall exergy balance, based on the definition of exergy property components before and after fission, is the following:

$$
\begin{aligned}
& \underset{\text { FISSION }}{\triangle E X_{\text {NUCLAR }}^{G}} \\
& =-\left[\left(\begin{array}{cc}
\text { NUCLEUS } \\
\text { BINDING } \\
\left.0+0+M^{\text {ENERGY }} \eta^{N}\right)^{\text {FISSION }}
\end{array}\right]\right.
\end{aligned}
$$

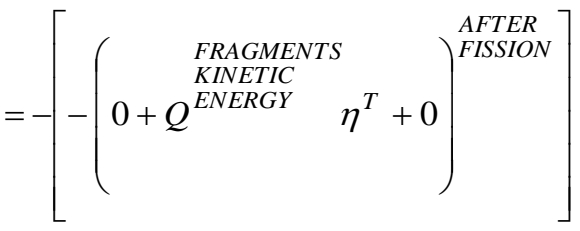

In case of nucleus fission, $\eta^{T}$ and $\eta^{N}$ are the efficiencies of ideal direct conversion cycles operating between the reservoir and the system thermodynamic conditions corresponding to kinetic energy and the potential energy of fission fragments. Interactions in the above equation can be expressed in terms of energy transfer associated to interacting particles before and after fission:

$$
\begin{aligned}
& \triangle E X_{\text {NUCLEAR }}^{G} \\
& \text { FISSION } \\
& =-\left[\left(0+0-E^{\text {NINDING }} \eta^{N}\right)^{\text {BEFSIRE }}\right] \\
& -\left[-\left(0-E^{\text {FRAGMETICNTS }} \eta^{T}+0\right)^{\text {AFTER }} \text { FISSION }\right]
\end{aligned}
$$

The Qvalue of a nuclear fission is about $210 \mathrm{MeV}$ and it is calculated with the measurement of mass defect of nuclei before and after fission [20]. The Qvalue is partitioned in the following contributions (in $M e V$ ): kinetic energy of fission fragments $=170$; kinetic energy of prompt neutron $=$ 10 ; prompt $\gamma=10$. Moreover, as concerns the decay of fission fragments: kinetic energy of delayed neutrons = negligible; energy associated to $\beta^{-}=5$; energy associated to delayed $\gamma=5$; energy associated to neutrinos $\bar{v}=10$ taking into account that neutrinos (generated by $\beta^{-}$decay) are not recovered and their energy is irreversibly lost. In addition to the mass defect, equal to the amount of thermal energy release and the associated thermal entropy contribution, nuclear fissions also determine a subdivision of the initial nucleus mass into fragments hence implying thermal entropy production due to inherently stochastic distribution of fragments velocities. Considering that the mechanical exergy is null before and after fission, then the calculation of generalized thermodynamic entropy for nuclear fission results from the following expression:

$$
\begin{aligned}
& \Delta S_{\text {FISSION }}^{G}=\left(S_{1}-S_{0}\right)^{T}+\left(S_{1}-S_{0}\right)^{N} \\
& =\frac{1}{T_{R}}\left[\left(E_{1}-E_{0}\right)-\left(E X_{1}^{R}-E X_{0}^{R}\right)\right]^{T} \\
& +\frac{1}{\mu_{R}^{N}}\left[\left(E_{1}-E_{0}\right)-\left(E X_{1}^{R}-E X_{0}^{R}\right)\right]^{N}
\end{aligned}
$$

As the reservoir is an auxiliary external reference system, its thermodynamic state can be arbitrarily selected to simplify the calculation. It can be assumed that reservoir conditions are those existing before the fission occurs. Then, temperature and nuclear potential of the reservoir are: $T_{R}=$ $298 \mathrm{~K}$ corresponding to the environmental initial temperature of target nucleus and neutron before fission; $\mu_{R}^{N}=\mu_{1}^{N}=-\begin{gathered}\text { NUCLEUS } \\ \text { BINDING } \\ \text { FRAGMENTS }\end{gathered} / n_{1}=1785 / 4,43 \cong 403 \mathrm{MeV}$

is the nuclear potential corresponding to the ratio of binding potential energy over the number $n_{1}$ of fragments after fission, with minus sign. Once the fission has occurred, then for the selected thermodynamic state of the reservoir, the calculation of entropy change can be separated for thermal process and nuclear process.

As concerns the thermal entropy variation related to the reference temperature:

$$
\begin{aligned}
& \left(S_{1}-S_{0}\right)^{T}=\frac{1}{T_{R}}\left[\left(E_{1}-E_{0}\right)-\left(E X_{1}^{R}-E X_{0}^{R}\right)\right]^{T} \\
& =\frac{1}{298}\left[210-\left(200 \cdot\left(1-\frac{T_{R}}{T_{1}}\right)-0\right)\right]^{T}
\end{aligned}
$$

It should be noticed that the whole amount of Qvalue, released as thermal energy along the nucleus fission, can be assumed as subdivided among 2 fission fragments and 2,43 neutrons as a mean value, that is 4,43 particles generated from 2 initial interacting particles, namely neutron and target nucleus. Therefore, by virtue of the equipartition theorem of energy among all fission fragments, each particle has associated an amount of kinetic energy equal to $210 / 4,43 \cong 47,4 \mathrm{MeV}$ constituting the thermal potential to be accounted for: 


$$
\begin{aligned}
& \left(S_{1}-S_{0}\right)^{T} \\
& =\frac{1}{298}\left[210-\left(200 \cdot\left(1-\frac{0,025 \cdot 10^{-6}}{47,4}\right)-0\right)\right]^{T}
\end{aligned}
$$

\section{$\cong 0,0336 \mathrm{MeV} / \mathrm{K}$}

where the heat-to-work conversion efficiency has been assumed as $\eta^{T} \cong 1$ being the final fission temperature far much higher than the initial reference temperature: hence, the energy released during fission can be considered equal to the thermal exergy content and the increase of thermal entropy is due to the kinetic energy associated to lost neutrinos.

As concerns the nuclear entropy variation related to the reference nuclear potential:

$$
\begin{aligned}
& \left(S_{1}-S_{0}\right)^{N} \\
& =\frac{1}{\mu_{R}^{N}}\left[\left(E_{1}^{\text {FRAGDINGNTS }}-E_{0}^{\text {NUINDINES }}\right)\right]^{N} \\
& +\frac{1}{\mu_{R}^{N}}\left[-\left(E_{1}^{\text {FRAGDING }}\left(1-\frac{\mu_{R}^{N}}{\mu_{1}^{N}}\right)\right)\right]^{N} \\
& +\frac{1}{\mu_{R}^{N}}\left[-\left(E_{0}^{\text {BINDING }}\left(1-\frac{\mu_{R}^{N}}{\mu_{0}^{N}}\right)\right)\right]^{N}
\end{aligned}
$$

where binding energies before fission and after fission are those calculated by means of the mass defect as reported in the literature [18]; nuclear potentials account for the number of particles, hence $\mu_{0}^{N}=1985 \mathrm{MeV}$ and $\mu_{1}^{N}=\mu_{R}^{N}=403 \mathrm{MeV}$ as assumed, thus:

$$
\begin{aligned}
& \left(S_{1}-S_{0}\right)^{N} \\
& =\frac{1}{403}\left[(1785-1985)-\left(0-1985 \cdot\left(1-\frac{403}{1985}\right)\right)\right]^{N} \\
& \cong 3,4 \mathrm{MeV} / \mathrm{K}
\end{aligned}
$$

The positive increase pertaining to both thermal and nuclear components of entropy variation above calculated confirms that a fission reaction determines an increase of fragments velocities associated to the velocity distribution and position dispersion caused by the increase of the number of particles generated from a unique nucleus.

Finally, the variation of the generalized thermodynamic entropy resulting from the sum of its components is:

$$
\begin{aligned}
& \Delta S_{\text {FISSION }}^{G}=\left(S_{1}-S_{0}\right)^{T}+\left(S_{1}-S_{0}\right)^{N} \\
& \cong 0,0336 \mathrm{MeV} / \mathrm{K}+3,4 \mathrm{MeV} / \mathrm{K} \\
& \cong 3,4336 \mathrm{MeV} / \mathrm{K}
\end{aligned}
$$

The amount of Qvalue is available as thermal energy and transferred to the cooling medium assumed at $573 \mathrm{~K}$ (typical average temperature of the coolant in a Light Water
Reactor) by means of heat interaction. In terms of thermal exergy, this implies that the maximum net useful work is derived considering the amount of heat interaction converted into work interaction by a reversible process between the fragments temperature and the cooling medium temperature. However, the temperature corresponding to $E^{\text {FRAGETENTS }}$ is much higher, therefore the thermal exergy between fission temperature and cooling medium temperature is irreversibly lost. The Qvalue is the amount of inter-fragments repulsion potential energy $E^{\text {REPULSION }}$ transformed into interfragments kinetic energy $E^{\text {FRAGETIC }}$, resulting from the nucleus fission, that can be finally withdrawn by the cooling medium from the temperature of fuel rods. However, an additional amount is recovered from structures and the coolant itself. Hence, the difference between the initial binding energy of the nucleus and the Qvalue of the fission process represents the amount of available energy irreversibly dissipated, corresponding to the exergy destruction, along the process starting from a unique system and ending to a set of fragments. The generalized exergy balance is:

$$
\begin{aligned}
& \underset{\text { FISSION }}{\triangle E X_{\text {NULLAR }}^{G}} \\
& =-\left[\left(\begin{array}{c}
\text { NUCLEUS } \\
\left.-E^{\text {BINDING }} \eta^{N}\right)^{\text {BISSION }}
\end{array}\right]\right.
\end{aligned}
$$

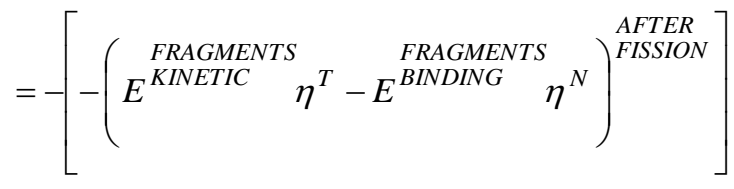

As the difference of thermodynamic potentials between nuclear fuel and external environment (reservoir) is high, then $\eta^{N} \cong 1$ can be assumed, therefore:

$$
\begin{aligned}
& \Delta E X_{\text {NUCLEAR }}^{G} \\
& =-\left[(-1785)-\left(200 \cdot\left(1-\frac{298}{573}\right)-1985\right)\right] \\
& =-[-1785-200 \cdot 0,48+1985]=-104 \mathrm{MeV} V
\end{aligned}
$$

The above amount of generalized exergy decrease $\underset{\text { FISSION }}{\Delta E X_{\text {NULAR }}^{G}}=-104 \mathrm{MeV}$ expresses the available mechanical exergy $E X^{M}$ irreversible destruction along the fission process of a single neutron and target nucleus. The reason of irreversibility implying exergy destruction is that a fraction of the initial binding energy of the whole nucleus is irreversibly dissipated because of: i) the subdivision of the nucleus into three or more fragments determining a change from high density energy to low density energy, and ii) the lower temperature, assumed at $573 \mathrm{~K}$, of Qvalue heat-towork conversion with respect to the fission temperature that makes the available energy, immediately after the fission, as a quasi-exergy. Finally, considering the definition of exergy 
efficiency $\eta^{E X}=1-E X_{D E S T R} / E X_{I N P U T}$, the equation relating to an elemental fission reaction of ${ }_{92} U^{235}$ is:

$$
\eta_{\text {FISSION }}^{\text {EX }}=1-\Delta E X_{\text {DESTR }}^{\text {NUCLEAR }} / \Delta X_{\text {AVAILABLE }}^{\text {NUCLEUS }}
$$

$=1-(200-104) / 200=0,52$

The enhancement of fission nuclear plants overall efficiency can be achieved by means of optimized plant configurations such us cogeneration to produce process heating in addition to the generation of electric power [21].

\section{Fusion Nuclear Reaction Exergy Analysis}

A typical fusion nuclear reaction occurs through the following process [22-23]:

$$
{ }_{1} H^{2}+{ }_{1} H^{3} \rightarrow{ }_{2} \mathrm{He}^{4}(3,5 \mathrm{MeV})+{ }_{0} n^{1}(14,1 \mathrm{MeV})
$$

where ${ }_{1} H^{2}$ and ${ }_{1} H^{3}$ are isotopes of hydrogen, Deuterium (D) and Tritium (T) respectively. Thermodynamic conditions to allow this reaction are generated inside the plasma chamber where electro-magnetic field are suitable to confine charged particles. Differently from fission, the fusion process is generated by colliding nuclei with an initial kinetic energy far much higher than the inter-nuclei kinetic energy corresponding to the environment temperature; those nuclei move along the same direction and opposite way to ensure that the collision occurs. This additional nuclei's kinetic energy is needed to overcome the electro-magnetic repulsion potential energy acting before short-radius strong interactions among nucleons prevail. After the fusion, the system results to be more stable with a higher binding potential energy implying a release of energy consisting of the Qvalue $=17,594 \mathrm{MeV}$ representing the net useful available energy transferred to the external system. The Qvalue corresponds to the thermal energy used by the heat transfer system conveying this thermal interaction to be converted into work interaction in turn released to be utilized by the external useful system. Fusion results from colliding ${ }_{1} H^{2}$ and ${ }_{1} H^{3}$ nuclei and the subsequent transmutation into ${ }_{2} \mathrm{He}^{4}$ with higher atomic number and by-production of one neutron. An increase of reaction products kinetic energy, due to the fusion process and corresponding to the Qvalue, occurs. The amount of Qvalue is calculated with the measurement of mass defect of nuclei before and after fusion [22]. Differently from fission, nuclear fusion creates an aggregation of the initial nuclei masses into one unique nucleus hence implying thermal entropy decrease due to the annihilation of the stochastic distribution of initial nuclei velocities.

The exergy balance of a nuclear fusion can be stated as follows:

$$
\underset{\substack{\text { FUSION } \\ \text { FUCLAR }}}{G}=\Delta E X^{M}+\Delta E X^{T}+\Delta E X^{N}
$$

$=-\left[\left(E X^{M}+E X^{T}+E X^{N}\right)^{\text {BEFORION }}\right]$
$-\left[-\left(E X^{M}+E X^{T}+E X^{N}\right)^{\text {FESIOR }}\right]$

Before the collision occurs, Deuterium and Tritium nuclei undergo an increase of velocity along the same direction and opposite ways needed to provide the thermodynamic conditions for nuclei fusion. To do so, an amount of work interaction input is used to increase the kinetic energy $E^{\text {KIIELTIC }}$ of nuclei up to a range of $116 \cdot 10^{6} \mathrm{~K}$ to $1160 \cdot 10^{6} \mathrm{~K}$ corresponding to a range of $10 \mathrm{keV}$ to 100 $\mathrm{keV}$. This work interaction is associated to the mechanical

exergy $E X^{M}=Q^{\substack{\text { NICLEI } \\ \text { ENERGY }}}$ calculated in terms of maximum net useful heat output resulting in the transfer of thermal energy associated to the temperature of colliding nuclei. Instead, the work interaction associated to the mechanical exergy NUCLEI

$E X^{M}=M^{\text {POTERGY }}$ calculated in terms of maximum net useful mass, is negligible because both strong and electro-magnetic interactions are negligible at the distance between particles NUCLEI before the collision. The thermal exergy $E X^{T}=W^{\text {KINETIC }}$ relating to the temperature of nuclei equal to the environment temperature before entering the confined plasma can be NUCLEI
POTENTIAL considered null. The nuclear exergy $E X^{N}=W^{\text {POTERGIIAL }}$ of

Deuterium and Tritium is null as the binding potential energy NUCLEI $E^{\text {BINDING }}$ of individual interacting nuclei is equal to the sum of ${ }_{1} H^{2}=2,224 \mathrm{MeV}$ and ${ }_{1} H^{3}=8,482 \mathrm{MeV}$ thus it couldn't be converted into maximum net useful work being in a more stable configuration with respect to isolated protons and neutrons as reported in the Figure 1.

After the collision has occurred, products velocity is distributed over random directions so that are characterized by products kinetic energy $E^{\text {PRODUCTIS }}$ determining the thermal energy being transferred as heat interaction output PRODUCTS KINETIC

$Q^{E N E R G Y}$ to the external system by means of the first wall,

the breading blanket and divertors constituting the main components of the primary heat transfer system from plasma chamber of a fusion reactor. The binding potential energy

$E^{\text {PRODINING }}$ of ${ }_{2} \mathrm{He}^{4}$ formed after collision is equal to 28,3 $\mathrm{MeV}$ representing the mass interaction output $M^{\begin{array}{c}\text { PRODUCTEST } \\ \text { POTENTIAL }\end{array}}$.

The mechanical exergy $E X^{M}$ after the fusion process is null since the whole content of energy is released under the form of thermal energy. Based on these contributions, the exergy balance is the following: 


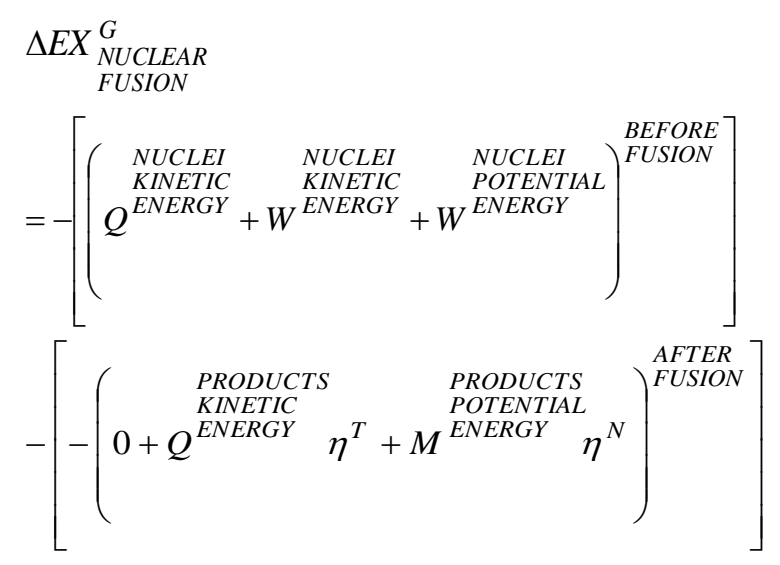

that can be expressed as:

$$
\begin{aligned}
& \begin{array}{c}
\Delta E X_{\text {NUCLEAR }}^{G} \\
\text { FUSION }
\end{array} \\
& =-\left[\left(\begin{array}{c}
\text { NUCLEI } \\
\left.E^{\text {KINETIC }} \eta^{T}+0+E^{\text {NUCLEIENTIAL }} \eta^{N}\right)^{\text {FESIORE }}
\end{array}\right]\right. \\
& -\left[-\left(0+E^{\text {PRODUCTS }} \eta^{\text {KINETIC }} \eta^{\text {PRODUCTS }} \begin{array}{c}
\text { AFTER } \\
\text { POTENTIAL }
\end{array} \eta^{N}\right)^{\text {FUSION }}\right]
\end{aligned}
$$

It is noteworthy that, before fusion, the attraction potential energy between Deuterium and Tritium due to strong interactions $E^{\text {NUCLENEIIAL }}$ is negligible. Instead, after fusion, the importance of attraction strong interactions potential energy becomes prevailing and provides an additional contribution to the products kinetic energy that is to be accounted for in the Qvalue. In terms of exergy balance, the attraction potential energy could not be transferred as useful work thus implying that it does not contribute to the exergy balance so the difference between before and after only contributes

The calculation of generalized thermodynamic entropy for nuclear fusion thus results in the following expression:

$$
\begin{aligned}
& \Delta S_{\text {FUSION }}^{G}=\left(S_{1}-S_{0}\right)^{T}+\left(S_{1}-S_{0}\right)^{N} \\
& =\frac{1}{T_{R}}\left[\left(E_{1}-E_{0}\right)-\left(E X_{1}^{R}-E X_{0}^{R}\right)\right]^{T} \\
& +\frac{1}{\mu_{R}^{N}}\left[\left(E_{1}-E_{0}\right)-\left(E X_{1}^{R}-E X_{0}^{R}\right)\right]^{N}
\end{aligned}
$$

As concerns the thermal entropy variation related to the temperature, assuming, also in this case, that $T_{R}$ corresponds to the environmental initial temperature of target nucleus and neutron before fusion, then:

$$
\left(S_{1}-S_{0}\right)^{T}=\frac{1}{T_{R}}\left[\left(E_{1}-E_{0}\right)-\left(E X_{1}^{R}-E X_{0}^{R}\right)\right]^{T}
$$

In the special case of fusion reaction, $T_{R}$ can be assumed equal to the temperature of ${ }_{1} H^{2}$ and ${ }_{1} H^{3}$ before nuclei collision, that is:
$T_{R}=10 \mathrm{keV}=0,01 \mathrm{MeV}=116 \cdot 10^{6} \mathrm{~K}$, so that:

$$
\begin{aligned}
& \left(S_{1}-S_{0}\right)^{T} \\
& =\frac{1}{116 \cdot 10^{6}}\left[17,594-\left(17,594 \cdot\left(1-\frac{T_{R}}{T_{1}}\right)+0,01 \cdot\left(1-\frac{T_{R}}{T_{0}}\right)\right)\right]
\end{aligned}
$$

that, using the numerical values becomes:

$$
\begin{aligned}
& =\frac{1}{116 \cdot 10^{6}}\left[17,594-\left(17,594 \cdot\left(1-\frac{0,01}{17,594}\right)+0,01 \cdot\left(1-\frac{0,01}{0,01}\right)\right)\right] \\
& \cong \frac{1}{116 \cdot 10^{6}}(-17,594-17,594 \cdot 0.9994) \\
& \cong 9,1 \cdot 10^{-5} \cdot 10^{-6} \mathrm{MeV} / \mathrm{K}
\end{aligned}
$$

This result demonstrates that the variation of thermal entropy along a nuclear fusion is negligible by virtue of the high temperature of products before and after the collision and nuclei aggregation has occurred. Though, the even negligible increase of thermal entropy reveals that the available energy, or the Qvalue, is released under the subdivided thermal form of energy associated to the thermal entropy positive variation. It is noteworthy that this variation is substantially lower with respect to the case of nuclear fission. Indeed, the rationale behind this difference is twofold: i) fission implies increase of particles as fragments of the initial target nucleus while fusion does not since it creates two products, Helium and neutron, from two reactants, Deuterium and Tritium; ii) the Helium nucleus has an atomic number, and therefore a mass number, higher than Deuterium or Tritium hence implying a higher mass concentration in one nucleus thus determining a decrease of thermal entropy associated to an increase of kinetic energy density.

As far as the nuclear entropy variation related to the nuclear potential is concerned, the following expression applies:

$\left(S_{1}-S_{0}\right)^{N}=\frac{1}{\mu_{R}^{N}}\left[\left(E_{1}-E_{0}\right)-\left(E X_{1}^{R}-E X_{0}^{R}\right)\right]^{N}$

where $\mu_{R}^{N}$ can be assumed equal to the nuclear potential of ${ }_{1} H^{2}$ and ${ }_{1} H^{3}$ before nuclei collision, that is $\mu_{R}^{N}=2,224 M e V+8,482 M e V=10,706 M e V$, then the previous expression is as follows:

$$
\begin{aligned}
& \left(S_{1}-S_{0}\right)^{N}=\frac{1}{\mu_{R}^{N}}\left\{\left(E_{1}-E_{0}\right)\right\} \\
& +\frac{1}{\mu_{R}^{N}}\left\{-\left[\left(\mu_{1}^{N} n_{1}^{N}\right) \cdot\left(1-\frac{\mu_{1}^{N}}{\mu_{1}^{N}}\right)-\left(\mu_{0}^{N} n_{0}^{N}\right) \cdot\left(1-\frac{\mu_{1}^{N}}{\mu_{0}^{N}}\right)\right]\right\}
\end{aligned}
$$

that, using the numerical values, becomes:

$$
\begin{aligned}
& =\frac{1}{10,706}\left[(10,706-28,3)-\left(10,706 \cdot\left(1-\frac{10,706}{10,706}\right)-28,3 \cdot\left(1-\frac{10,706}{28,3}\right)\right)\right] \\
& =\frac{1}{10,706}[(-17,594)-(-17,594)]=0 \mathrm{MeV} / \mathrm{K}
\end{aligned}
$$


In this case, the variation of nuclear entropy is null since there is no variation of the number of particles between the initial and final states of the reaction: thus, from a physical standpoint, there is no change in the degree of subdivision of the binding energy content before and after releasing the Qvalue. Moreover, it has been assumed that, for two interacting particles, the nuclear potential $\mu_{R}^{N}$ equals the potential energy of the system hence the whole amount of potential energy is transferred as work interaction to the useful external system. Instead, from three interacting particles on, the nuclear potential would progressively decrease as the progressive increase of the number of particles is accounted for. Hence, the variation of nuclear entropy becomes positive because of the not complete transfer of nuclear potential into work interaction in the respect of Second Law.

The number of particles before and after the reaction is conserved although mass aggregation is not, hence the variation of entropy depends on both distribution of mass and velocity caused by the collision and the consequent fusion process.

The variation of exergy property, calculated before and after ${ }_{1} H^{2}$ and ${ }_{1} H^{3}$ nuclei collision, can be assumed as the maximum net useful interaction expressing the definition of generalized exergy. Therefore:

$$
\begin{aligned}
& -\Delta E X_{\text {FUCLEAR }}^{G}=\left(0,1 \eta^{T}+0+2,224 \eta^{N}+8,482 \eta^{N}\right)^{\text {FUSION }} \\
& -\left(0-17,594 \eta^{T}+28,3 \eta^{N}\right)^{\text {FUSEION }} \\
& -\Delta E X_{\text {FUCLEAR }}^{G}=6,274 \mathrm{MeV}
\end{aligned}
$$

where it is assumed that $\eta^{N} \cong 1$ from plasma chamber fusion temperature to blanket temperature assumed $823 \mathrm{~K}$ and $\eta^{T}=\left(1-\frac{298}{823}\right)=0,638$ from blanket to environment temperature at $298 \mathrm{~K}$, then a decrease of exergy occurs in fusion process equal to $\underset{ }{\triangle E X_{\text {NUCLEAR }}^{G}} \cong-6,274 \mathrm{MeV}$.

The physical meaning of this result is that almost the entire amount of energy released along a nuclear fusion process constitutes the available energy, in terms of generalized exergy, deriving from the decrease of mass subsequent to the increase of inter-nucleon potential energy with respect to the isolated nucleons before fusion. The decrease of exergy is accompanied by a decrease of entropy due to a different configuration of the whole system in which an increase of level in the hierarchical structure is due to the mass fusion ${ }_{1} H^{2}+{ }_{1} H^{3} \rightarrow{ }_{2} H e^{4}+n$. Indeed, free hydrogen isotopes result in a bind system of higher mass. To do so, a fraction of initial nuclei kinetic energy $E^{\text {KINETIC }}$ is adsorbed during fusion reaction. Then, the fusion process is irreversible due to the destruction of a part of initial exergy content that does not completely compensate the entropy reduction due to fusion of D and T nuclei.

Finally, the exergy efficiency is:

$$
\begin{aligned}
& \eta_{\text {FUSION }}^{E X}=1-\Delta E X_{\text {DESTR }}^{\begin{array}{c}
\text { NUCLEAR } \\
\text { FUSION }
\end{array}} / \Delta E X_{\text {AVAILABLE }}^{\text {NUCLEI }} \\
& =1-(17,594-11,22) / 17,594=0,638
\end{aligned}
$$

This is a theoretical result that is to be reduced taking into account the real processes occurring in the plasma chamber of a fusion reactor.

\section{Conclusions}

Two main conclusions can be outlined from the present research. Firstly, the model of few-particle thermodynamic systems is adopted to determine all phenomena and properties, involved in nuclear fission and fusion processes. This investigation has been carried out in the perspective of the Second Law analysis based on the exergy method adopted to calculate entropy property variations. The second result concerns the consequence of decrease or increase of nuclei atomic number and mass number subsequent to elemental fission and fusion nuclear reactions and therefore the subdivision or aggregation of atomic nuclei. The role of entropy property, in these special cases, is to quantify the impact on energy and exergy balances, along nuclear fission or fusion processes, responsible of the different behavior of a few-particle system. A conclusive consideration arising from the aforementioned results is that nuclear physics and physics of elementary particles undergo non-equilibrium processes. In this regard, irreversibilities determined by nonequilibrium states and processes are governed by extrema principles, rooted on entropy property variations, such as the maximum entropy production principle [24], representing an innovative contribution to analysis methods and researches in those fields.

\section{Nomenclature}

$A$ : internal system

$C: \quad$ constant parameter

$E$ : energy $\mathrm{J} / \mathrm{mol}$

$E X^{G}$ : generalized exergy $\mathrm{J} / \mathrm{mol}$

$E X^{M}$ : mechanical exergy $\mathrm{J} / \mathrm{mol}$

$E X^{N}$ : nuclear exergy $\mathrm{J} / \mathrm{mol}$

$E X^{T}$ : thermal exergy $\mathrm{J} / \mathrm{mol}$

$M$ : mass interaction $\mathrm{J} / \mathrm{mol}$

$n: \quad$ mol

$P$ : $\quad$ pressure $\mathrm{Pa}$

$Q$ : heat interaction $\mathrm{J} / \mathrm{mol}$

$R$ : reservoir

$\bar{R}$ : $\quad$ universal constant $\mathrm{J} / \mathrm{mol} \mathrm{K}$

$S^{G}$ : generalized entropy $\mathrm{J} / \mathrm{mol} \mathrm{K}$

$S^{M}$ : mechanical entropy $\mathrm{J} / \mathrm{mol} \mathrm{K}$

$S^{N}: \quad$ nuclear entropy $\mathrm{J} / \mathrm{mol} \mathrm{K}$

$S^{T}: \quad$ thermal entropy $\mathrm{J} / \mathrm{mol} \mathrm{K}$

$T: \quad$ temperature $\mathrm{Pa}$

$U: \quad$ internal energy $\mathrm{J} / \mathrm{mol}$ 


$$
\begin{array}{ll}
V: & \text { volume } m^{3} \\
W: & \text { work interaction } \mathrm{J} / \mathrm{mol} \\
\mu: & \text { nuclear potential } \mathrm{J} / \mathrm{mol} \\
\eta: & \text { efficiency }
\end{array}
$$

\section{References}

[1] W.R. Dunbar, S.D. Moody and N. Lior, "Exergy Analysis of an Operating Boiling-Water-Reactor Nuclear Power Station," Energy Conversion and Management, 36, 3, 149-159, 1995. doi:10.1016/0196-8904(94)00054-4.

[2] A. Durmayaz, H. Yavuz, "Exergy Analysis of a Pressurized-Water Reaction Nuclear Power Plant," $J$. Appl. Energy, 69, 39, 2001.

[3] L. Ferroni, A. Natale and R. Gatto, "Exergy Analysis of a PWR Core Heat Transfer," International Journal of Heat and Technology, 34, 2, S465-S472, 2016, doi: https://doi.org/10.18280/ijht.34S239.

[4] V. Badescu, D. Isvoranu, "Classical Statistical Thermodynamics Approach for the Exergy of Nuclear Radiation," Europhys. Lett. 80, 30003, 2007.

[5] V. Badescu, "Exergy of Nuclear Radiation - a Quantum Statistical Thermodynamics Approach," Cent. Eur. J. Phys., 7, 141, 2009.

[6] W.A. Hermann, "Quantifying Global Exergy Resources,” Energy, 31, 1685-1702, 2006.

[7] E. Gyftopoulos, G.P. Beretta, Thermodynamics: Foundations and Applications, New York: Dover Publications, 2005.

[8] E. Gyftopoulos, “An Inherent, Non-statistical Property of any System in any State," Int. J. of Thermodynamics, 9, 3, 107-115, 2006.

[9] G.P. Beretta, "Axiomatic Definition of Entropy for Nonequilibrium States," Int. J. Thermodynamics, 11, 2, 39-48, 2008.

[10] E. Zanchini, G.P. Beretta, "Removing Heat and Conceptual Loops from the Definition of Entropy," Int. J. Thermodynamics, 13, 2, 67-76, 2010.

[11] G.P. Beretta, E. Zanchini, "A Definition of Thermodynamic Entropy Valid for Non-equilibrium States and Few-particle Systems," arXiv 2014;1411.5395.

[12] P. Palazzo, "Theorem of Necessity and Sufficiency of Stable Equilibrium for Generalized Potential Equality between System and Reservoir," J. Modern Physics, 5, 2003-2011, 2014.

[13] P. Palazzo, "A Method to Derive the Definition of Generalized Entropy from Generalized Exergy for Any State in Many-Particle Systems," Entropy, 17, 20252038, 2015, doi:10.3390/e17042025.

[14] P. Palazzo, "A Generalized Statement of HighestEntropy Principle for Stable Equilibrium and NonEquilibrium in Many-Particle Systems," J. Modern Physics, 7, 344-357, 2016.

[15] W.R. Dunbar, N. Lior and R.A. Gaggioli, "The Component Equations of Energy and Exergy," J. Energy Resources Technology, 114, 1992.

[16] R.A. Gaggioli, "Available Energy and Exergy," International Journal of Applied Thermodynamics, 1, 14, 1-8, 1998

[17] R.A. Gaggioli, D.H. Richardson and A.J. Bowman, "Available Energy - Part I: Gibbs Revisited," J. Energy Resources Technology, June 2002.

[18] R.A. Gaggioli, D.M. Jr. Paulus, “Available Energy Part II: Gibbs Extended," Transaction of the ASME 2002, June.

[19] T.J. Kotas, The Exergy Method of Thermal Plant Analysis, Reprint edn, Krieger Publishing Company, 1995.

[20] S. Glasstone , M.C. Edlund, The Elements of Nuclear Reactor Theory, $5^{\text {th }}$ Printing, Princeton, New Jersey: D. Van Nostrand Company, Inc., 1952.

[21] Z. Dong, Y. Pan, "A lumped-parameter dynamical model of a nuclear heating reactor cogeneration plant," Energy, 145, 638-656, 15 February 2018, doi.org/10.1016/j.energy.2017.12.153.

[22] W.M. Stacey, Fusion: An Introduction to the Physics and Technology of Magnetic Confinement Fusion, $2^{\text {nd }}$ Edition, WILEY-VCH, 2010. ISBN:978-3-527-40967-9.

[23] J.I. Linares, A. Cantizano, B.Y. Moratilla, V. MartinPalacios and L. Batet, "Supercritical CO2 Brayton Power Cycles for DEMO (demonstration power plant) Fusion Reactor Based on Dual Coolant Lithium Lead Blanket," Energy, 98, 271-283, 1 March 2016, doi.org/10.1016/j.energy.2016.01.020.

[24] L.M. Martyushev, V.D. Seleznev, "Maximum Entropy Production Principle in Physics, Chemistry and Biology," Phys. Rep., 426, 1-45, 2006, doi:10.1016/j.physrep.2005.12.001. 